\title{
REGULAR CLOSED MAPS
}

\author{
R. F. DICKMAN, JR.
}

\begin{abstract}
A subset $A$ of $X$ is far from the remainder if whenever $\mathscr{U}$ is a free open ultrafilter in $X$ there exists $U \in \mathscr{U}$ such that $A \cap \mathrm{cl}_{X} U=\varnothing$. A map is regular closed provided that the image of every regular closed set is closed. In this note we use some recent results of $\mathrm{G}$. Viglino to show that every map can be extended to a regular closed map with far from the remainder point inverses. We also relate these maps to several other interesting classes of maps.
\end{abstract}

Introduction. Throughout this paper all spaces are assumed to be Hausdorff and all functions are assumed to be continuous. A subset $A$ of a space $X$ is a regular closed subset of $X$ if $A=\operatorname{cl}_{X}$ (int $A$ ) and a map $f: X \rightarrow Y$ is a regular closed map if for every regular closed set $A \subset X, f(A)$ is closed in $Y$. A subset $A$ of $X$ is said to be far from the remainder (f.f.r.) if for every free open ultrafilter $\mathscr{U}$ in $X$, there exists $V \in \mathscr{U}$ such that $\operatorname{cl}_{X} V \cap A=\varnothing$.

An extension of a map $f: X \rightarrow Y$ is a map $F: Z \rightarrow Y$ such that $X$ is a proper dense subset of $Z$ and $F \mid X=f$ and we say that a space $X$ is $f$-absolutely closed (alternately $f$ is absolutely closed) provided that no extension of $f: X \rightarrow Y$ exists. In [4] G. Viglino gave some characterizations of absolutely closed maps. In this note we give another such characterization and relate these maps to several interesting classes of maps.

\section{A characterization.}

TheOREM 1 [4, TheOREM 1.2]. A map $f: X \rightarrow Y$ is absolutely closed provided that whenever $\mathscr{U}$ is an open filter in $X$ such that $f(\mathscr{U})$ converges in $Y$, $\mathscr{U}$ has a nonempty adherent set in $X$.

THEOREM 2. A map $f: X \rightarrow Y$ is absolutely closed if and only if it is regular closed and point inverses are f.f.r.

Proof of The NeCESSITy. Let $A$ be a regular closed subset of $X$ and suppose that $y \in \operatorname{cl}_{Y} f(A) \backslash f(A)$. Let $\mathscr{N}(y)$ denote the open neighborhood filter of $y$. Then for every $N \in \mathscr{N}(y), f^{-1}(N) \cap A$ is nonempty as is $f^{-1}(N) \cap$ int $A$. Thus $\left\{f^{-1}(N) \cap\right.$ int $\left.A: N \in \mathscr{N}(y)\right\}$ is a base for an open filter $\mathscr{U}$ such

Presented to the Society, November 25, 1972; received by the editors September 22, 1972.

AMS (MOS) subject classifications (1970). Primary 54C10; Secondary 54D25.

Key words and phrases. Regular closed maps, absolutely closed maps.

(c) American Mathematical Society 1973 
that $f(\mathscr{U})$ converges to $y$ in $Y$. By Theorem $1, \operatorname{ad}_{X} \mathscr{U}$, the adherent set of $\mathscr{U}$ in $X$, is a nonempty subset of $f^{-1}(y)$. But if $x \in \operatorname{ad}_{X} \mathscr{U}, x$ belongs to $A$ and so $f(x)=y \in f(A)$. This contradiction implies that $f$ is regular closed.

In order to see that point inverses are f.f.r. let $y \in Y$ and let $\mathscr{U}$ be a free open ultrafilter. Then by Theorem 1 and the maximality of $\mathscr{U}, y \notin \operatorname{ad}_{Y} f(\mathscr{U})$. Thus there exists an open set $V$ containing $y$ and $U \in \mathscr{U}$ such that $V \cap$ $f(U)=\varnothing$. Then $f^{-1}(V) \cap U=\varnothing$ and $f^{-1}(y) \cap \mathrm{cl}_{X} U=\varnothing$. Thus $f^{-1}(y)$ is f.f.r.

ThE SUFFICIENCY. Suppose that $\mathscr{U}$ is a free open filter in $X$ and suppose that $f(\mathscr{U})$ converges to $y \in Y$. Since $X$ is a regular closed set, $f(X)$ is a closed subset of $Y$ so that $y \in f(X)$. By our hypothesis $f^{-1}(y)$ is f.f.r., hence there exists $U \in \mathscr{U}$ such that $f^{-1}(y) \cap \mathrm{cl}_{X} U=\varnothing$. But then $A=\operatorname{cl}_{X} U$ is regular closed and $f(A)$ is a closed set not containing $y$. This is impossible since $Y \backslash f(A)$ would then be a neighborhood of $y$ containing no $V \in \mathscr{U}$. This contradiction implies that $f$ is absolutely closed.

COROllary 2.1. Every map can be extended to a regular closed map with f.f.r. point inverses.

This follows immediately from Theorem 2 and Theorem (2.2) of [4].

REMARKS. The following question is posed in [4]: Is every closed map with point inverses $H$-closed relative to $X$ an absolutely closed map? We have seen that replacing the term "closed map" in the question with the weaker term "regular closed map", and strengthening the condition "point inverses are $H$-closed relative to $X$ " to "point inverses are f.f.r." gives a characterization of absolutely closed maps. The question in [4] may then be rephrased as follows: If point inverses of a closed map are $H$-closed relative to $X$, are they also f.f.r.? One can easily show that if point inverses of a map are f.f.r., they are also $H$-closed relative to $X$. There are $H$-closed subsets which are not f.f.r. See [1, p. 48].

Let $\tau X$ denote the Katětov $H$-closed extension of $X$ [3]. A map $f: X \rightarrow Y$ is called $\tau$-proper provided there exists a map $\tau f: \tau X \rightarrow \tau Y$ such that $\left.\tau f\right|_{X}=f$ and a $\tau$-proper map is said to be $\tau$-perfect if $\tau f(\tau X \backslash X) \subset(\tau Y \backslash Y)$. In [1, Theorem 5] A. Blaszczyk and J. Mioduszewski showed that every $\tau$-perfect map is absolutely closed. Every open map is $\tau$-proper [1] so $\tau$-proper maps are not necessarily absolutely closed. The example of $[1$, p. 49] shows that absolutely closed maps are not necessarily $\tau$-proper. By Theorem 2 and Theorem 3 of [1], a $\tau$-proper map is absolutely closed if and only if it is $\tau$-perfect.

THEOREM 3. Every $\tau$-proper map $f: X \rightarrow Y$ can be extended to a $\tau$-perfect map.

Proof. Let $Z=(\tau f)^{-1}(Y)$ and $g=\tau f \mid Z$. Then $\tau Z$ is an absolute closure of $X$ and by [3, Theorem (1.13)] there exists a map $h: \tau X \rightarrow \tau Z$ such that 
$h \mid X$ is the identity. Also $\tau X$ is an absolute closure of $Z$ so there exists a map $g: \tau Z \rightarrow \tau X$ such that $g \mid Z$ is the identity. Thus $h$ and $g$ are inverses and $\tau X$ is the Katětov extension of $Z$. Since $g$ can be extended to $\tau X$, and $g(\tau X \backslash X) \subset$ $(\tau Y \backslash Y), g$ is $\tau$-perfect as required.

Remarks. The author has been able to show that every map can be extended in a unique manner to a perfect map, however the extension often behaves badly in that the domain usually fails to be a Hausdorff space [2]. One would expect this to be the case since a nonperfect map on an absolutely closed space cannot be extended to a perfect map on a Hausdorff space. Since every map on an absolutely closed space is regular closed and every set is f.f.r. we avoid this difficulty in the above extension.

\section{REFFRENCES}

1. A. Blaszczyk and J. Mioduszewski, On factorization of maps through $\tau X$, Colloq. Math. 23 (1971), 45-52.

2. R. F. Dickman, Jr., On closed extensions of functions, Proc. Nat. Acad. Sci. U.S.A. 62 (1969), 326-332. MR 42 \#5233.

3. C. T. Liu, Absolutely closed spaces, Trans. Amer. Math. Soc. 130 (1968), 86-104.

4. G. Viglino, Extensions of functions and spaces, Trans. Amer. Math. Soc. (to appear).

Department of Mathematics, Virginia Polytechnic Institute and State UNIVERSITY, BLACKSBURG, VIRGINIA 24061 